\title{
Combined Quantitative Analysis Using both Micro-XRF and EDS Analysis Inside the Scanning Electron Microscope
}

\author{
Kenny C. Witherspoon, Rich Lamb, Per Sjoman, and Mandi D. Hellested, MS FIS
}

IXRF Systems, Inc., 3019 Alvin Devane, Suite 130, Austin, Texas, 78741 USA

In 2004 [1], the first commercial Micro-focus x-rays tubes were added to the Scanning Electron Microscope (SEM) providing x-ray fluorescence (XRF). These x-ray tubes excite a sample and produce characteristic X-rays in the same manner as Energy Dispersive Spectroscopy (EDS). The characteristic X-rays are then collected by an unmodified EDS detector. The addition of XRF compliments standard EDS analysis primarily due to the absence of the background continuum created by the decelerated electrons generated by the electron beam (Bremsstrahlung). The electron beam is more suited for lighter elements, below $2.0 \mathrm{keV}$. While XRF analysis typically detects ppm level trace elements above $2.0 \mathrm{keV}$, hence offering an increased level of analytical capability over standard EDS systems.

Since 2004, advancements in several areas have been made with the addition of XRF in the SEM. The $\mathrm{x}$-ray sources have gone through several iterations to enhance performance. One such iteration is the improvements of focusing apertures and the amount of power, which causes an increase in flux. Micro-focus tubes require a very small excitation area on the anode of the tube, which in turn creates a much "brighter" spot moving the x-rays through the focusing optics. Also geometries from earlier designs of x-ray sources have been improved through mechanical advancements. Specimen holders and sample preparation have gone through advancements to optimize the analysis for both excitations. [2].

An even more important advancement is combining micro-XRF and EDS in the SEM [3]. The two techniques are an ideal complement for elemental analysis. This compliment can be seen in Fig.1, which is a combined EDS/XRF analysis highlighting each elements excitation interaction with a given standard .The electron beam excites the lighter elements more efficiently and the XRF beam excites higher energy elements more efficiently. The ability of XRF to excite higher energies also allows for better peak separation, which in turn allows for better peak identification. Fig. 2 is a spectral overlay of a metal sample, which exhibits the ability of microXRF to identify elements that are not seen with electron excitation alone, but to also resolve peaks that would otherwise be lost to background. The micro-XRF spectrum is able to resolve the calcium K-alpha peak, which is lost within the background of the EDS. With EDS, the zirconium L-lines and lead M-lines overlap at the low energy region and it appears as though molybdenum might be present as well. However, micro-XRF resolves the L-lines of lead as well as the $\mathrm{K}$-alpha and $\mathrm{K}$-beta lines of zirconium, but does not show molybdenum $\mathrm{K}$ lines.

The combination of micro-XRF and EDS is not only a useful identification technique, but it can also be used as a quantification technique. In Fig.1 "Combined Analysis", first the EDS spectrum is acquired in the top of the dual panel, the electron beam is then shut off, so as not to contaminate the micro-XRF spectrum with increased background, then the Micro-XRF spectrum is then collected in the lower panel. The two spectra are analyzed with both ZAF for EDS and FP (fundamental parameters) for XRF. This allows for nearly all elements in a given sample to be identified and analyzed with a single technique. Once the two spectra have been acquired, a variety of software algorithms can be applied. Each element can be assigned to a specific excitation, lighter elements for the electron beam and the heavier elements to the XRF. One quantitative routine called "Auto" automatically selects which element will be best analyzed with which quantitative routine given its specific excitation. Ideally, standards would be used for both 
excitations to calibrate the system to better analyze the unknowns. Another analysis technique the employs the dual panel is Least Squares using linear regressions. This allows for the use of multiple standards in a single file. This approach also allows for multiple standards to be used for individual elements since each element can be defined independently.

Furthermore, when comparing bench top XRF spectra to spectra from XRF inside the SEM, increased sensitivity for elements below $2.0 \mathrm{keV}$ can be seen. This is due to the SEM environment providing a very high vacuum atmosphere as compared to bench top XRF units. Also, most EDS detectors for SEM have either "windowless" detectors or thin polymer windows allowing for greater transmittance of the lighter element X-rays versus Be window XRF benchtop units.

[1] Cross, B.J. and Witherspoon, K.C., Microscopy \& Microanalysis 10-2 (2004) 104-105.

2. Cross, B.J. and Augenstine, J.E., Adv. X-ray Analysis 34 (1991) 57-70.

3 Cross, B.J. and Witherspoon, K.C., Proc. IMC16, Sapporo, (2006) 879.

4 - Cross, B. and Witherspoon, K.C., "XRF in the SEM-How and Why?" presented at $52^{\text {nd }}$ Denver X-Ray Conference (2003).
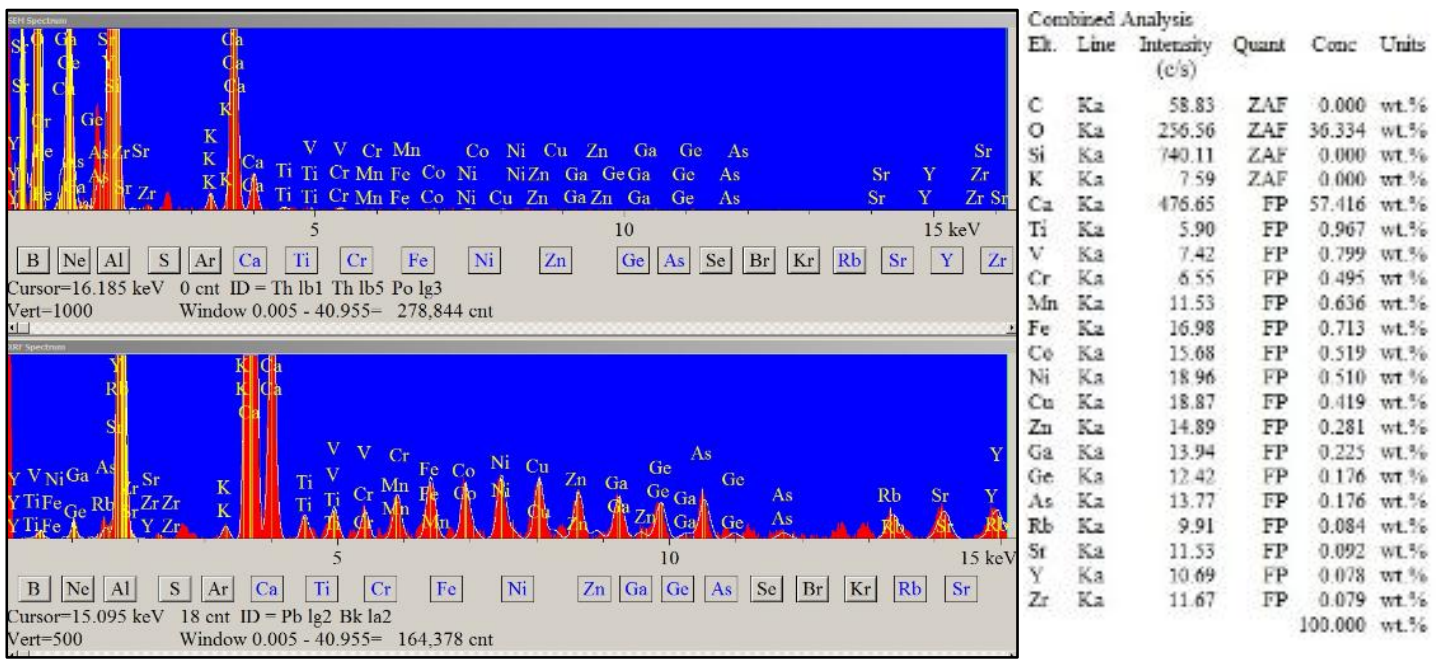

Fig. 1. Combined Analysis panel EDS specturm on top and XRFspectrum on bottom, of NIST 610 Glass Standard

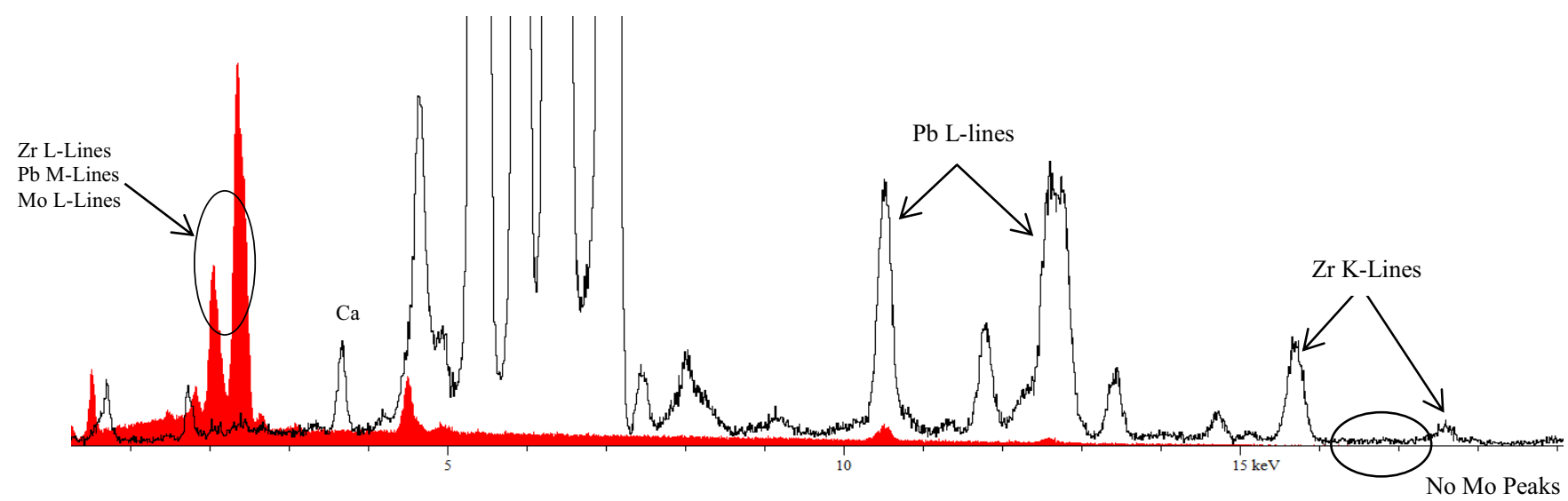

Fig. 2. Spectral overlay of EDS and XRF metal sample 